\title{
SCATTERING OF ANTIPLANE WAVES BY INHOMOGENEOUS ALLUVIAL VALLEYS
}

\author{
By Yoshiji NIWA* and Sohichi HIROSE**
}

\begin{abstract}
Scattering problems of antiplane waves incident upon inhomogeneous alluvial valleys are studied by using the integral equation methods. Three types of integral equation approaches are presented and compared each other. The first one is the boundary-domain integral equation based on the static fundamental solution for a homogeneous body. The second one is identical to the first one, except that the dynamic fundamental solution is employed. The last one is the boundary integral equation for the scatterer with ad hoc inhomogeneity, for which the fundamental solution is obtained. The numerical results show that the soft surface layer in alluvial valleys induces the large amplification and long duration of surface motions, which reflects the important problem in earthquake engineering.
\end{abstract}

\section{INTRODUCTION}

Topografical and geological irregularities have much effect on seismic motions, which have been investigated by several methods, e.g., perturbation method, FDM (finite difference method), FEM (finite element method), ray theory, discrete wave number method, BIEM (boundary integral equation method), and so forth. Among these methods, nowadays, the BIEM has been well developed in elastodynamics, and applied to various wave propagation problems in a half space, since the BIEM is manageable to deal with the problem in a half space directly ${ }^{11}$.

Many authors applied BIEM to scattering problems of antiplane (SH) waves due to surface irregularities, such as canyons ${ }^{2)}{ }^{4)}$ and slopes $^{5)}$, and due to geological irregularities, such as alluvial valleys $^{(6)}$ and inclusions ${ }^{12)}$. For scattering problems of plane (P-SV) waves in a half space, many studies were carried out on surface topography ${ }^{13)}$ and on geological irregularities ${ }^{5), 10)}{ }^{12), 14)}$. Axisymmetrical irregularities in a three-dimensional half space were also investigated by Sánchez-Sesma ${ }^{15}$.

In all these analyses, the scatterers themselves consist of homogeneous materials which are different from those in a surrounding half space. From the practical point of view, however, it may be necessary to refer to geological irregularities as inhomogeneous materials. Several methods were proposed for the analyses of inhomogeneous bodies. For example, Kobayashi \& Kishima ${ }^{16)}$ applied the hybrid method of boundary integral equation and finite element to dynamic analyses of non-homogeneous ground. Niwa et al. ${ }^{17) .21)}$ used the boundary-domain integral equation method for the dynamic analyses of inhomogeneous structures. In this paper, three approaches of integral equation methods are presented in order to analyze surface motions in inhomogeneous alluvial valleys subjected to incident $\mathrm{SH}$ waves.

* Member of JSCE, Dr. Eng., Professor, Department of Civil Engineering, Kyoto University (Sakyo-ku, Kyoto 606)

** Member of JSCE, M. Eng., Instructor, Department of Civil Engineering, Kyoto University 


\section{STATEMENT OF PROBLEM}

Let us consider the antiplane wave motion in the two-dimensional model as shown in Fig. 1. The region $D^{i}$ is an inhomogeneous alluvial valley of arbitrary shape, which is perfectly bonded to the homogeneous half-space $D^{e}$ on the boundary $\partial M$. It should be noted that the material properties in domain $D^{i}$ are not homogeneous, but inhomogeneous from the assumption. The incident field $v^{I}$ is assumed to be the plane harmonic $\mathrm{SH}$ wave with the incident angle $\alpha$. Under the above assumptions, the governing equations in domains $D^{i}$ and $D^{e}$ are written as

$$
\left(\mu^{i}(\underset{\sim}{X}) u_{, j}(\underset{\sim}{X}, \omega)\right)_{, j}+\rho^{i}(\underset{\sim}{X}) \omega^{2} u(\underset{\sim}{X}, \omega)=0 \quad \underset{\sim}{X} \text { in } D^{i}
$$

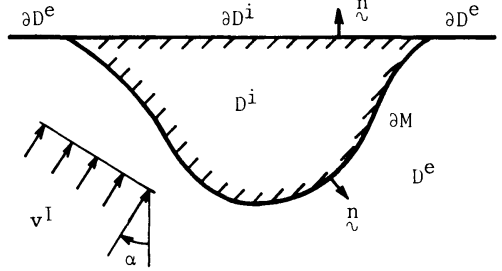

Fig. 1 Geometry of alluvial valley in a half space.

$$
\mu^{e} v_{, j j}(\underset{\sim}{X}, \omega)+\rho^{e} \omega^{2} v(\underset{\sim}{X}, \omega)=0 \quad \underset{\sim}{X} \text { in } D^{e}
$$

where $u$ and $v$ are the antiplane fields in domain $D^{i}$ and domain $D^{e}$, respectively. $\mu^{i}$ and $\rho^{i}\left(\mu^{e}\right.$ and $\left.\rho^{e}\right)$ are the shear modulus and mass density in domain $D^{i}$ (domain $D^{e}$ ). We use the conventional notation ${ }_{, j}$ for $\partial / \partial X_{j}$ and $\omega$ is the angular frequency.

The boundary conditions on boundaries $\partial D^{i}$ and $\partial D^{e}$, and the continuity condition on boundary $\partial M$ are specified as

$$
\begin{aligned}
& t(\underset{\sim}{x}, \omega) \equiv T\left(\mu^{i}(\underset{\sim}{x}), \underset{\sim}{n} ; \partial\right) u(\underset{\sim}{x}, \omega)=0 \quad \underset{\sim}{x} \text { on } \partial D^{i} \\
& s(\underset{x}{x} \omega) \equiv T\left(\mu^{e}, \underline{n} ; \partial\right) v(\underset{x}{x}, \omega)=0 \quad x \text { on } \partial D^{e} \text {. } \\
& u(\underset{x}{x}, \omega)=v(\underset{x}{x}, \omega)
\end{aligned}
$$

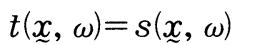

$$
\begin{aligned}
& \underline{x} \text { on } \partial M
\end{aligned}
$$

where $\underline{n}$ is the normal vector to the boundary and $T(\mu, \underline{n} ; \partial)$ is the traction operator defined as

$T(\mu, \underset{\sim}{n} ; \partial) \equiv \mu n_{j} \partial_{j}$.

Furthermore, the scattered field $v^{s}=v-v^{F}$ in domain $D^{e}$ is assumed to satisfy the radiation conditions

$$
\partial v^{s} / \partial r-i \omega v^{s} / C_{T}^{e}=o\left(r^{-\frac{1}{2}}\right), \quad v^{s}=o\left(r^{\frac{1}{2}}\right)
$$

when $r \equiv|\underset{\sim}{X}| \rightarrow \infty$, where $v^{F}=v^{I}+v^{R}$ ( $v^{R}$ indicates the reflected field in the case of the absence of the alluvial valley) and $C_{T}^{e}$ is the velocity of $\mathrm{SH}$ wave defined as

$$
C_{T}^{e}=\sqrt{\mu^{e} / \rho^{e}} \text {. }
$$

The problem is to find the solution $u(\underset{\sim}{X}, \omega)$ of eqs. (1) and (2), subjected to the boundary and continuity conditions (3) and (4), and the radiation condition (5). In the case that the transient wave field $\hat{u}(\underset{\sim}{X}, t)$ is required, the following inverse Fourier transform is available,

$$
\hat{u}(\underset{X}{X}, t)=\frac{1}{2 \pi} \int_{-\infty}^{\infty} u(\underset{X}{X}, \omega) e^{-i \omega t} d \omega .
$$

\section{FORMULATION OF INTEGRAL EQUATIONS}

\section{(1) Integral equations for the interior domain $\boldsymbol{D}^{i}$}

Three types of integral representations for the interior domain $D^{i}$ are presented. In the first and second subsections, static and dynamic fundamental solutions for homogeneous bodies are used in order to derive the boundary-domain integral equations for a general inhomogeneous medium. In the last subsection, we consider the alluvial valley with ad hoc inhomogeneity, for which the fundamental solution is obtained in the explicit form. In this case, the boundary integral equation can be formulated even if the material in $D^{i}$ has the inhomogeneity. Hereafter, the methods presented in the subsections a), b) and c) are referred to as Methods $\mathrm{A}, \mathrm{B}$ and $\mathrm{C}$, respectively.

a) Method based on the static fundamental solution (Method A)

Here, we separate the shear modulus $\mu(\underset{\sim}{X})\left(\underset{\sim}{X}\right.$ in $\left.D^{i}\right)$ into the constant part $\mu^{\dagger}$ and the variable part $\delta \mu(\underset{\sim}{X})$ 
as follows,

$$
\mu^{i}(\underset{\sim}{X})=\mu^{\dagger}+\delta \mu(\underset{\sim}{X}) \quad \underset{\sim}{X} \text { in } D^{i}
$$

Substituting eq. ( 7 ) into eq. (1), then we obtain

$$
\mu^{\dagger} u_{, j}(\underset{\sim}{X}, \omega)=-\left\{\left(\delta \mu(\underset{\sim}{X}) u_{, j}(\underset{\sim}{X}, \omega)\right)_{, j}+\rho^{i}(\underset{\sim}{X}) \omega^{2} u(\underset{\sim}{X}, \omega)\right\} \quad \underset{\sim}{X} \text { in } D^{i} .
$$

The right-hand side in the above equation, which is related to the material inhomogeneity and inertia force, is considered as the equivalent body force in the static problem. Consequently, the static fundamental solution $U^{\dagger}(\underset{Y}{X}, \underline{Y})$ for a homogeneous body is introduced,

$$
\mu^{\dagger} U_{, j j}^{\dagger}(\underset{\sim}{X}, \underset{\sim}{Y})=-\delta(\underset{\sim}{X}-\underset{\sim}{Y})
$$

where $\delta(\underset{\sim}{X}-\underset{\sim}{Y})$ denotes the Dirac measure. The fundamental solution $U^{\dagger}(\underset{\sim}{X}, \underset{\sim}{Y})$ is well known,

$$
U^{\dagger}(\underset{\sim}{X}, \underset{\sim}{Y})=\frac{1}{2 \pi \mu^{\dagger}} \ln \left(\frac{1}{r}\right)
$$

where $r \equiv|X-Y|$. From eqs. (8) and (9), the interior Green's formula has the following form,

$$
\begin{aligned}
& \int_{\partial D^{i}+\partial M} U^{\dagger}(\underset{\sim}{X}, \underset{\sim}{y}) T\left(\mu^{\dagger}, \underset{\sim}{n} ; \partial\right) u(\underset{\sim}{y}, \omega) d s_{y}-\int_{\partial D^{i}+\partial M} T\left(\mu^{\dagger}, \underset{\sim}{n} ; \partial_{y}\right) U^{\dagger}(\underset{\sim}{X}, \underset{\sim}{y}) u(\underset{\sim}{y}, \omega) d s_{y} \\
& \quad+\int_{D^{i}} U^{\dagger}(\underset{\sim}{X}, \underset{\sim}{y})\left\{\left(\delta \mu(y) u_{, j}(\underset{\sim}{y}, \omega)\right)_{j}+\rho^{i}(\underset{\sim}{y}) \omega^{2} u(\underset{\sim}{y}, \omega)\right\} d S_{y}=\left\{\begin{array}{cc}
u(\underset{\sim}{X}, \omega) & \underset{\sim}{X} \text { in } D^{i} \ldots \\
0 & \underset{\sim}{X}\left(D^{i}\right)^{c}
\end{array}\right.
\end{aligned}
$$

where $\left(D^{i}\right)^{c}$ indicates the complementary domain to $D^{i}$. Applying the divergence theorem to the surface integral on the left-hand side of eq. (11), we have the integral equation

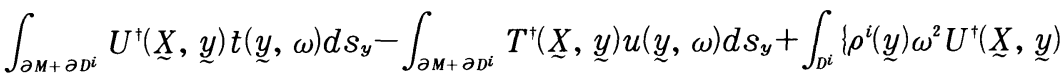

$$
\begin{aligned}
& \left.+\delta \mu_{, j}(\underset{\sim}{y}) U_{, y_{j}}^{\dagger}(\underset{\sim}{X}, \underset{v}{y})\right\} u(\underset{\sim}{y}, \omega) d S_{y}=\left\{\begin{array}{cc}
\mu^{i}(\underset{\sim}{X}) u(\underset{\sim}{X}, \omega) / \mu^{\dagger} & \underset{\sim}{X} \text { in } D^{i} \cdots \\
0 & \underset{\sim}{X} \text { in }\left(D^{i}\right)^{c}
\end{array}\right.
\end{aligned}
$$

where $T^{\dagger}(\underset{\sim}{X}, \underset{y}{)})$ is the kernel of double layer potential defined as

$$
T^{\dagger}(\underset{\sim}{X}, \underset{\sim}{y}) \equiv \widetilde{T}\left(\mu^{i}(\underset{\sim}{y}), \underset{\sim}{n} ; \partial_{y}\right) U^{\dagger}(\underset{\sim}{X}, \underset{\sim}{y})
$$

and , $y_{j}$ indicates $\partial / \partial y_{j}$. From the limiting procedure, $\underset{\sim}{X}$ in $\left(D^{i}\right)^{c}$ to the boundary $\partial D^{i}$ or $\partial M$, where $\underset{\sim}{x}$ is on $\partial D^{i}$ or $\partial M$, the boundary-domain integral equation is obtained as follows,

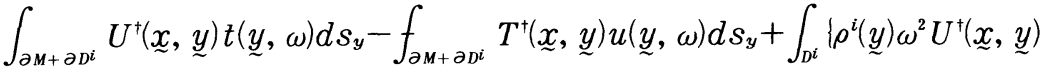

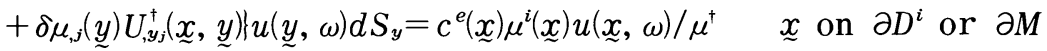

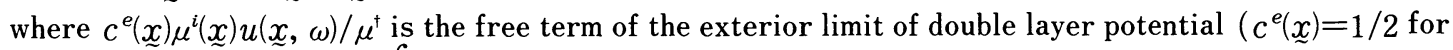
the smooth boundary), and $f \cdot d s$ denotes the principal value integral. The present formulation is identical to that in ref. 17).

b ) Method based on the dynamic fundamental solution (Method B)

In order to formulate the integral equation, we can utilize the dynamic fundamental solution $U^{*}(\underset{\sim}{X}, \underset{\sim}{Y})$ defined as a solution of the following differential equation,

$$
\mu^{*} U_{, j j}^{*}(\underset{\sim}{X}, \underset{\sim}{Y})+\rho^{*} \omega^{2} U^{*}(\underset{\sim}{X}, \underset{\sim}{Y})=-\delta(\underset{\sim}{X}-\underset{\sim}{Y})
$$

instead of the static one $U^{\dagger}(\underset{\sim}{X}, \underset{\sim}{Y})$. It is well known that $U^{*}(\underset{\sim}{X}, \underset{\sim}{Y})$ has the form

$$
U^{*}(\underset{\sim}{X}, \underset{\sim}{Y})=\frac{i}{4 \mu^{*}} H_{0}^{(1)}\left(k_{T}^{*} r\right)
$$

where $k_{T}^{*}=\omega\left(\mu^{*} / \rho^{*}\right)^{-1 / 2}$, and $H_{0}^{(1)}(\cdot)$ indicates the zeroth order Hankel function of the first kind. In this case, the shear modulus $\mu(\underset{\sim}{X})$ and mass density $\rho(\underset{\sim}{X})$ in $D^{i}$ are written as follow,

$$
\mu^{i}(\underset{\sim}{X})=\mu^{*}+\Delta \mu(\underset{\sim}{X}), \rho^{i}(\underset{\sim}{X})=\rho^{*}+\Delta \rho(\underset{\sim}{X}) \quad \underset{\sim}{X} \text { in } \tilde{D}^{i}
$$

where $\mu^{*}$ and $\rho^{*}$ are the arbitrary constants, and $\Delta \mu(\underset{\sim}{X})$ and $\Delta \rho(\underset{\sim}{X})$ are the deviations from $\mu^{*}$ and $\rho^{*}$, respectively. On the substitution of eq. (15) into eq. (1), we have

$$
\mu^{*} u_{, j j}(\underset{\sim}{X}, \omega)+\rho^{*} \omega^{2} u(\underset{\sim}{X}, \omega)=-\left\{\left(\Delta \mu(\underset{\sim}{X}) u_{, j}(\underset{\sim}{X}, \omega)\right)_{, j}+\Delta \rho(\underset{\sim}{X}) \omega^{2} u(\underset{\sim}{X}, \omega)\right\} \quad \underset{\sim}{X} \text { in } D^{i}
$$

From eqs. (13) and (16), the interior Green's formulae are expressed as

$$
\int_{\partial M+\partial D^{i}} U^{*}\left(\underset{\sim}{X}, \underset{v}{y} t(\underline{y}, \omega) d s_{y}-\int_{\partial M+\partial D^{i}} T^{*}(\underset{\sim}{X}, \underset{\sim}{y}) u(\underset{y}{y}, \omega) d s_{y}+\int_{D^{i}}\left\{\left(\Delta \rho(\underline{y})-\Delta \mu(\underline{y}) / \mu^{*}\right) \omega^{2} U^{*}(\underset{\sim}{X}, \underset{\sim}{y})\right.\right.
$$




$$
\begin{aligned}
& \left.+\Delta \mu_{, j}(\underset{\sim}{y}) U_{, y_{j}}^{*}(\underset{\sim}{X}, \underset{\sim}{y})\right\} u(\underset{\sim}{y}, \omega) d S_{y}=\left\{\begin{array}{cc}
\mu^{i}(\underset{\sim}{X}) u(\underset{\sim}{X}, \omega) / \mu^{*} & \underset{\sim}{X} \text { in } D^{i} \\
0 & \underset{\sim}{X} \text { in }\left(D^{i}\right)^{c}
\end{array}\right.
\end{aligned}
$$

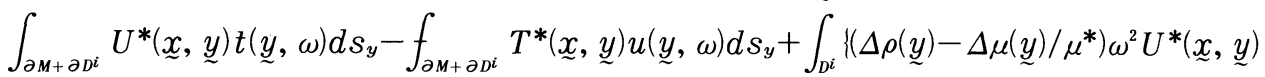

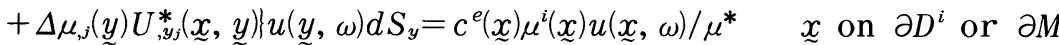

where $T^{*}(\underset{\sim}{X}, \underset{\sim}{y}) \equiv T\left(\mu^{i}(\underset{y}{y}), \underset{\sim}{n} ; \partial_{y}\right) U^{*}(\underset{\sim}{X}, \underset{y}{y})$. Eq. (17) is the counterpart of eq. (12) in the previous subsection.

c) Method for the ad hoc case (Method C)

Because of the difficulty in finding the fundamental solution for general inhomogeneous media, the well-known static and dynamic fundamental solutions are used in the before-mentioned formulations. In those cases, however, the domain integrals still remain (for example, see eqs. (12) and (17)), so that the region $D^{i}$ is needed to be discretized into body cells in numerical analysis. This subsection deals with the ad hoc inhomogeneous medium for which the fundamental solution is found.

It is assumed that the material properties in $D^{i}$ are given as

$$
\mu^{i}(\underset{\sim}{X}) / \mu^{0}=\left(p X_{2}+q\right)^{\gamma}, \quad \rho^{i}(\underset{\sim}{X}) / \rho^{0}=\left(p X_{2}+q\right)^{\gamma-2}
$$

where $\mu^{0}, \rho^{0}, p, q$ and $\gamma$ are constants, so that the wave velocity $C_{T}^{i}(\underset{\sim}{X})$ becomes the linear function with respect to $X_{2}$, i. e.,

$$
C_{T}^{i}(\underset{\sim}{X})=\sqrt{\mu^{i}(\underset{\sim}{X}) / \rho^{i}(\underset{\sim}{X})}=\left(p X_{2}+q\right) \sqrt{\mu^{0} / \rho^{0}} .
$$

On substitution of eq. (18) into eq. (1), the equation of motion is written in the form

$$
u_{, j j}(\underset{\sim}{X}, \omega)+\frac{\gamma p}{p X_{2}+q} u_{, 2}(\underset{\sim}{X}, \omega)+\frac{\left(k_{T}^{0}\right)^{2}}{\left(p X_{2}+q\right)^{2}} u(\underset{\sim}{X}, \omega)=0 \quad \underset{\sim}{X} \text { in } D^{i}
$$

where $k_{T}^{0}=\omega\left(\mu^{0} / \rho^{0}\right)^{-1 / 2}$. Here, we consider the fundamental solution $\Gamma$ which satisfies the following adjoint differential equation of eq. (19),

$$
\Gamma_{, j j}(\underset{\sim}{X}, \underset{\sim}{Y})-\left(\frac{\gamma p}{p X_{2}+q} \Gamma(\underset{\sim}{X} \underset{\sim}{Y})\right)_{, 2}+\frac{\left(k_{T}^{0}\right)^{2}}{\left(p X_{2}+q\right)^{2}} \Gamma(\underset{\sim}{X} \underset{\sim}{Y})=-\delta(\underset{\sim}{X}-\underset{\sim}{Y}) .
$$

The solution of eq. (20) is given in the following explicit form

$$
\Gamma(\underset{\sim}{X}, \underset{\sim}{Y})=\frac{1}{2 \pi}\left(\frac{g}{h}\right)^{\gamma / 2} Q_{\nu-1 / 2}\left(\frac{r^{2}}{2 g h}+1\right) \cdots
$$

where $Q_{\nu}(\cdot)$ denotes the Legendre function of the second kind, and $g, h$ and $\nu$ are defined as

$$
g=X_{2}+q / p, \quad h=Y_{2}+q / p, \quad \nu^{2}=((1-\gamma) / 2)^{2}-\left(k_{T}^{0}\right)^{2} / p^{2}
$$

respectively. The detailed derivation of $\Gamma(\underset{\sim}{X}, \underset{\sim}{Y})$ is referred to Appendix. From eqs. (19) and (20), we can obtain the boundary integral equation

$$
\begin{aligned}
& \int_{\partial D^{i}+\partial M} \Gamma(\underset{\sim}{y} \underset{\sim}{X}) \partial u(\underset{\sim}{y}, \omega) / \partial n d s_{y}-\int_{\partial D^{i}+\partial M} n_{j}(\underset{\sim}{)}) \Gamma_{, y_{j}}(\underset{\sim}{\underset{X}{X}} \underset{\sim}{X}) u(\underset{\sim}{y}, \omega) d s_{y} \\
& +\int_{\partial D^{i}+\partial M} n_{2}(\underset{\sim}{y}) \frac{\gamma p}{p y_{2+} q} \Gamma(\underset{\sim}{y} \underset{\sim}{X}) u(\underset{\sim}{y}, \omega) d s_{y}=\left\{\begin{array}{cc}
u(\underset{\sim}{X}, \omega) & \underset{\sim}{X} \text { in } D^{i} \\
0 & \underset{\sim}{X} \text { in }\left(D^{i}\right)^{c}
\end{array}\right. \\
& \int_{\partial D^{i}+\partial M} \Gamma(\underline{y}, \underset{x}{x}) \partial u(\underline{y}, \omega) / \partial n d s_{y}-f_{\partial D^{i}+\partial M} n_{j}(\underline{y}) \Gamma_{, y_{j}}(\underset{\sim}{y}, \underset{x}{x}) u(\underset{y}{y}, \omega) d s_{y} \\
& +\int_{\partial D^{i}+\partial M} n_{2}(\underset{\sim}{y}) \frac{\gamma p}{p y_{2}+q} \Gamma(\underset{\sim}{y}, \underset{\sim}{x}) u(\underset{y}{y}, \omega) d s_{y}=c^{e}(\underset{\sim}{x}) u(\underset{\sim}{x}, \omega) \quad \underline{x} \text { on } \partial D^{i} \text { or } \partial M .
\end{aligned}
$$

(2) Integral equation for the exterior domain $D^{e}$

Since $D^{e}$ is assumed to be a homogeneous half-space and the scattered field $v^{s}$ satisfies the radiation condition (5) at infinity, the conventional exterior Green's formulae are obtained,

$$
\begin{aligned}
& v^{F}(\underset{\sim}{X}, \omega)-\int_{\partial M} V^{e}(\underset{\sim}{X}, \underset{\sim}{y}) s(\underset{\sim}{y}, \omega) d s_{y}+\int_{\partial M} S^{e}(\underset{\sim}{X}, \underset{\sim}{y}) v(\underset{\sim}{y}, \omega) d s_{y} \\
& =\left\{\begin{array}{cc}
v(\underset{\sim}{X}, \omega) & \underset{\sim}{X} \text { in } D^{e} \text { or } \partial D^{e} \\
0 & \underset{\sim}{X} \text { in }\left(D^{e}\right)^{c} \ldots \ldots \ldots \ldots
\end{array}\right.
\end{aligned}
$$




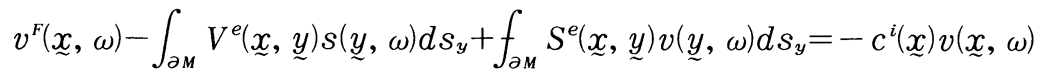

$$
\begin{aligned}
& x \text { on } \partial M
\end{aligned}
$$

where $V^{e}(\underset{\sim}{X}, \underset{\sim}{y})$ is the dynamic Green's function in a half-space and $S^{e}(\underset{\sim}{X}, \underset{y}{y}) \equiv T\left(\mu^{e}, \underset{\sim}{n} ; \partial_{y}\right) V^{e}(\underset{\sim}{X}, \underset{z}{y})$.

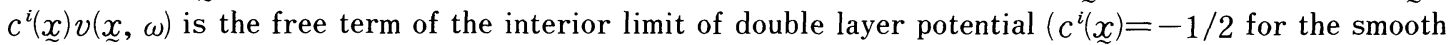
boundary). $V^{e}(\underset{\sim}{X}, \underset{\sim}{Y})$ has the well-known form

$$
V^{e}(\underset{\sim}{X}, \underset{\sim}{Y})=\frac{i}{4 \mu^{e}}\left(H_{0}^{(1)}\left(k_{T}^{e} r\right)+H_{0}^{(1)}\left(k_{T}^{e} r^{\prime}\right)\right)
$$

where $k_{T}^{e}=\omega\left(\mu^{e} / \rho^{e}\right)^{-1 / 2}$ and $r^{\prime} \equiv\left(\left(X_{1}-Y_{1}\right)^{2}+\left(X_{2}+Y_{2}\right)^{2}\right)^{1 / 2}$.

Taking account of the boundary condition (3) and the continuity condition (4), the integral equation (23) for the exterior domain is coupled with one of the integral equations (12), (17), and (22) for the interior domain, and solved numerically.

\section{NUMERICAL EXAMPLES}

\section{(1) Numerical remarks}

The alluvial valley considered here is assumed to be a semi-cylinder with the radius a as shown in Fig. 2 . We use 70 constant boundary elements and 625 constant triangular body cells. Since the integral kernels have singularities when $\underset{\sim}{X}=\underset{\sim}{Y}$, the analytical schemes $^{18)}$ are employed in order to evaluate the singular integrals. In the case of transient analyses, we make the following remarks on the procedure :

- The inverse Fourier transform is carried out by use of the fast Fourier transform (FFT) algorithm, where the frequen-

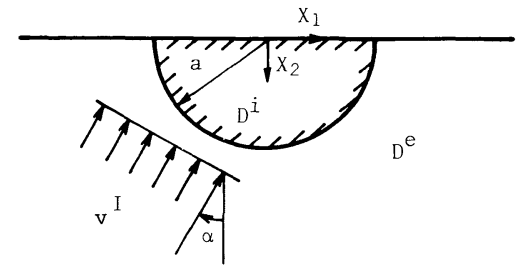

Fig. 2 The model of a semi-cylindrical alluvium. cies up to $a\left(k_{r}^{e}\right)_{\max }=2 \pi$ are taken into account.

- The Ricker wavelet $f(t)$ is used as the incident pulse,

$$
f(t)=2 A^{I}(\tau(t)-0.5) e^{-\tau(t)} \quad\left(\tau(t)=\left(C_{T}^{e} k_{T}^{p} t\right)^{2} / 4\right)
$$

where $A^{I}$ is the maximum amplitude of incident wave, and $k_{T}^{p}$ is the peak wave number. We choose $k_{T}^{p}$ as $k_{T}^{p}=\left(k_{T}^{e}\right)_{\max } / 2.5$.

- The maximum amplitude of the Ricker wavelet reaches the origin at time $t=0$.

\section{(2) Accuracy and comparison of our methods}

For a homogeneous and semi-cylindrical alluvial valley, the closed-form analytical solution was obtained by Trifunac ${ }^{19}$. Therefore, we compared our results with Trifunac's solution in order to confirm the accuracy of our methods. Figs. 3(a) and (b) show the displacement amplifications on the free surface for $a k_{T}^{e}=\pi$ and $2 \pi$, which were obtained by Method A. The material constants used here are given as

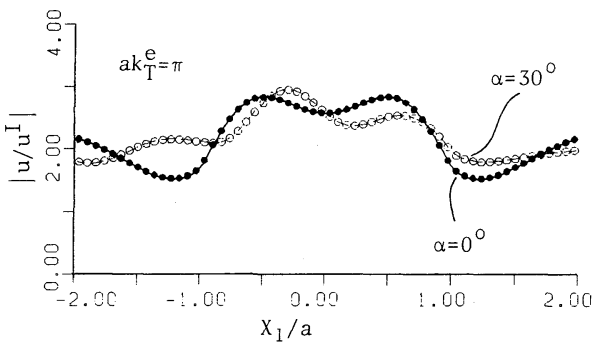

(a)

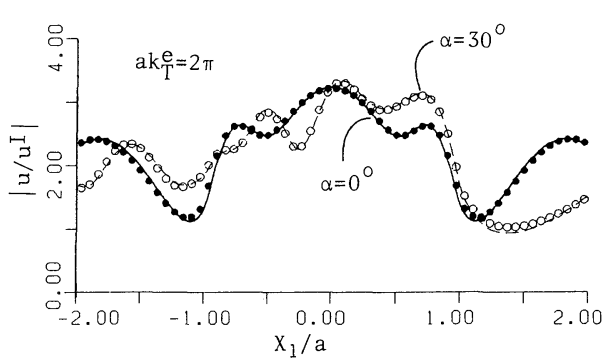

(b)

Fig. 3 Displacement amplitudes on the free surface in a steady state. 

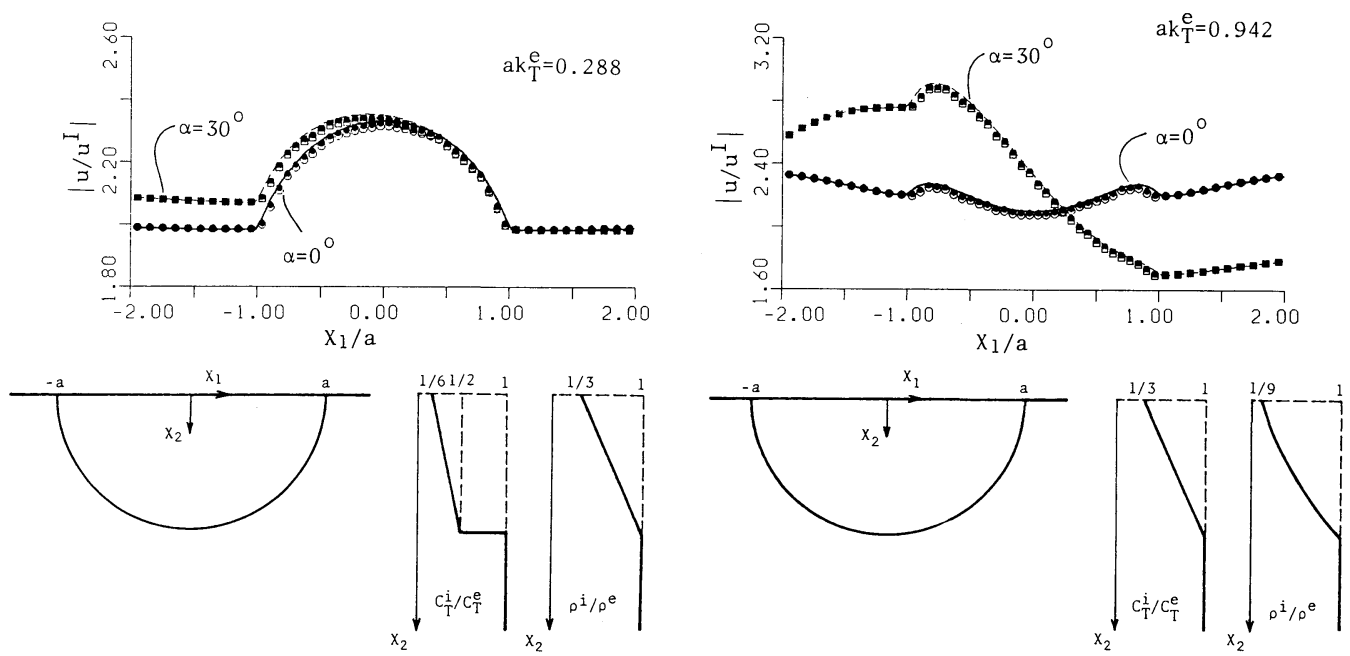

(a)

(b)

Fig. 4 Displacement amplitudes on the free surface in a steady state.

- $\square$ : Method A; $\bigcirc, \square$ : Method B;-—, ---: Method C.

Material properties in inhomogeneous alluvium are shown below them.

$\mu^{i} / \mu^{e}=0.5, \rho^{i} / \rho^{e}=1.0$.

Our results agree quite well with the analytical ones by Trifunac.

Figs. 4 (a) and (b) give the comparison among the displacement amplitudes on the free surface obtained by Methods A, B and C. The alluvial valley is assumed to be inhomogeneous with the material properties as shown directly below them. The results show a good agreement each other.

From Figs. 3 and 4, the accuracy of our methods is proved to be sufficient for the analysis of the seismic motions on the inhomogeneous ground.

The comparison among our three methods gives the following comments:

- Method B consumes more CPU time than Method A, because the dynamic fundamental solution (14) is more troublesome to deal with than the static one (10).

- Since the dynamic fundamental solution involves the angular frequency $\omega$ implicitly, Method B is inadequate to analyzing transient problems of seismic motions. On the other hand, the static fundamental solution in Method A is independent of the frequency $\omega$ and therefore is not necessary to be evaluated for each frequency.

- Since the unknowns in Method C are defined only on the boundary, Method C is the most feasible and efficient in numerical analysis. However, Method $\mathrm{C}$ has no generality because of the difficulty in obtaining a fundamental solution for a general inhomogeneous body.

From the above discussion it is concluded that Method A is the most suitable to wave analysis of inhomogeneous media. For this reason, we use Method A in the following analysis.

\section{(3) Surface motion in a steady-state}

Two types of semi-cylindrical alluvial valleys are considered,

inhomogeneous alluvium:

$\mu^{i} / \mu^{e}=0.8 X_{2} / a+0.1, \rho^{i} / \rho^{e}=1.0$

homogeneous alluvium :

$\mu^{i} / \mu^{e}=0.5, \rho^{i} / \rho^{e}=1.0$.

In both cases, the mean values of shear moduli over the depth of alluvial valley are the same, i. e., $a^{-1} \int_{0}^{a} \mu^{i} d X_{2}=0.5 \mu^{e}$. 


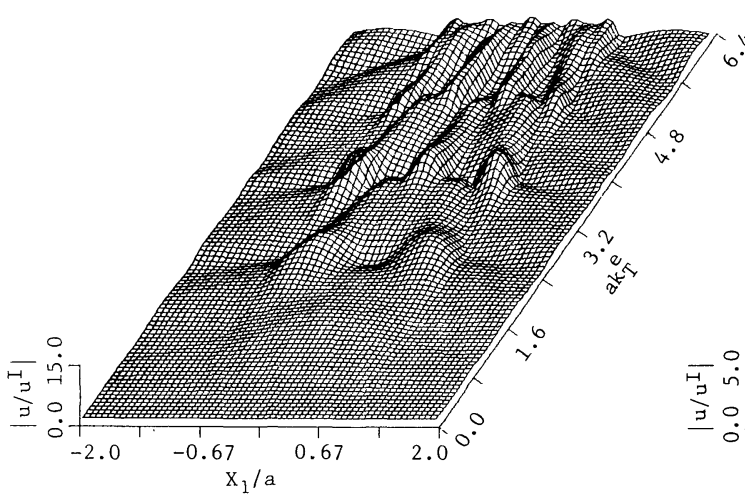

(a)

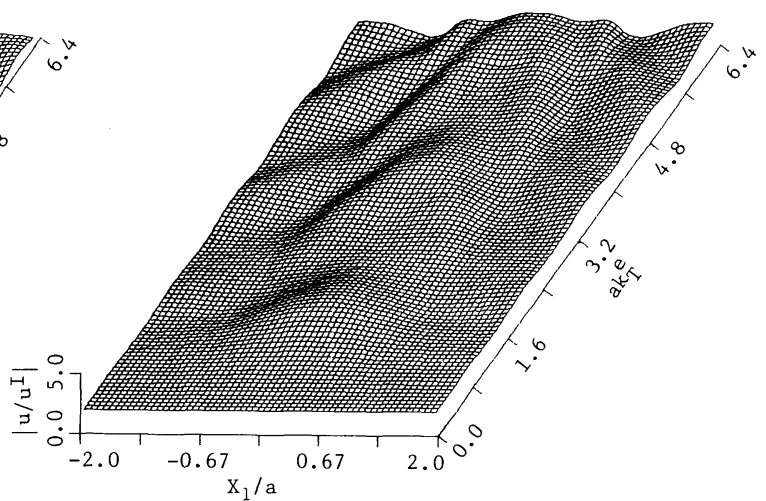

(b)

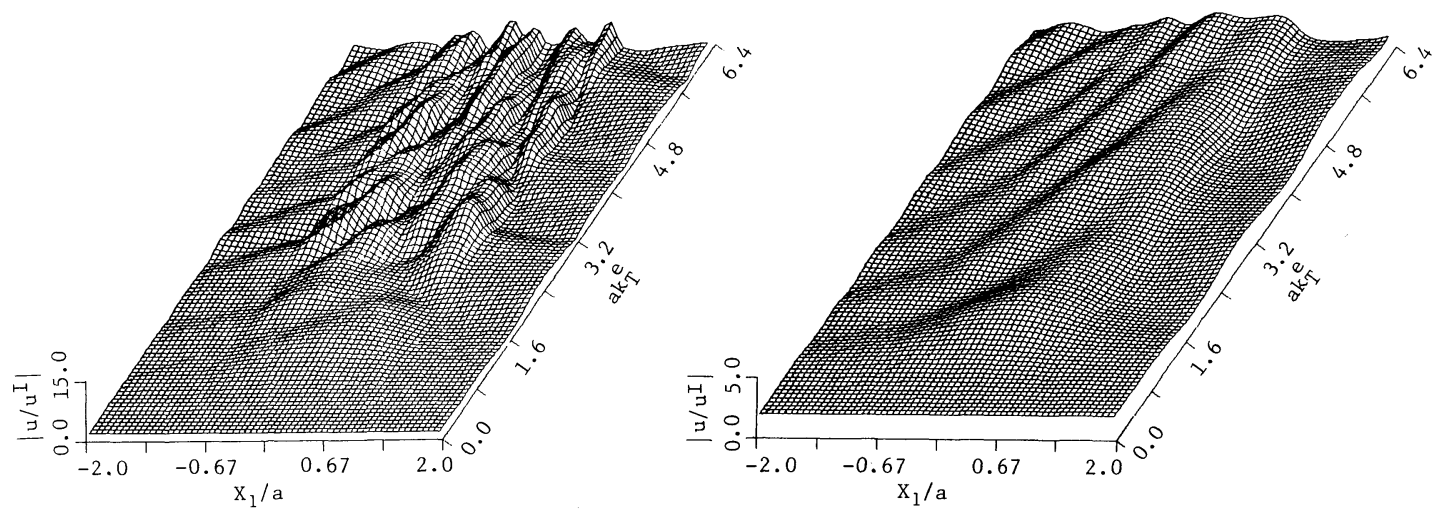

(c)

(d)

Fig. 5 Surface displacement amplitudes in a steady state for inhomogeneous alluvium ((a) and (c)) and homogeneous alluvium ((b) and (d)). Figs. (a) and (b) are for the vertical incidence of SH wave, and Figs. (c) and (d) for the oblique incidence with the angle $30^{\circ}$.

Figs. 5(a) and (b) show the displacement amplifications on the free surface in a steady-state for the vertical incidence of $\mathrm{SH}$ wave. The former is the case for the inhomogeneous alluvium and the latter is for the homogeneous one. The normalized wave numbers up to $a k_{T}^{e}=2 \pi$ are taken into account.

For the inhomogeneous valley, the complicated and large surface displacements are observed particularly in the high frequency range. On the other hand, for the homogeneous case, the pattern of amplification changes gradually from the lower mode to the higher one.

Figs. 5(c) and (d) show the surface displacements for the inhomogeneous and homogeneous valleys subjected to the oblique incident $\mathrm{SH}$ wave with the angle $\alpha=30^{\circ}$. The similar effect is observed as in the case of vertical incidence.

\section{(4) Transient surface motions}

The transient surface motions for the incidence of the Ricker SH wavelet are shown in Figs. 6 (a) to (d), whose models correspond to Figs. $5(\mathrm{a})$ to (d) in the previous section, respectively. In these figures, the displacement $\hat{u}(\underset{\sim}{X}, t)$ is normalized by the incident amplitude $A^{l}$ and the dimensionless time $t C_{T}^{e} / a$ is considered.

For the homogeneous valleys, only the small secondary reflected wave follows the primary wave, and then immediately, the surface motion attenuates. While, for the inhomogeneous ones, the primary wave shows the large amplitude on the free surface of the valley. After the passage of the primary wave, we can 

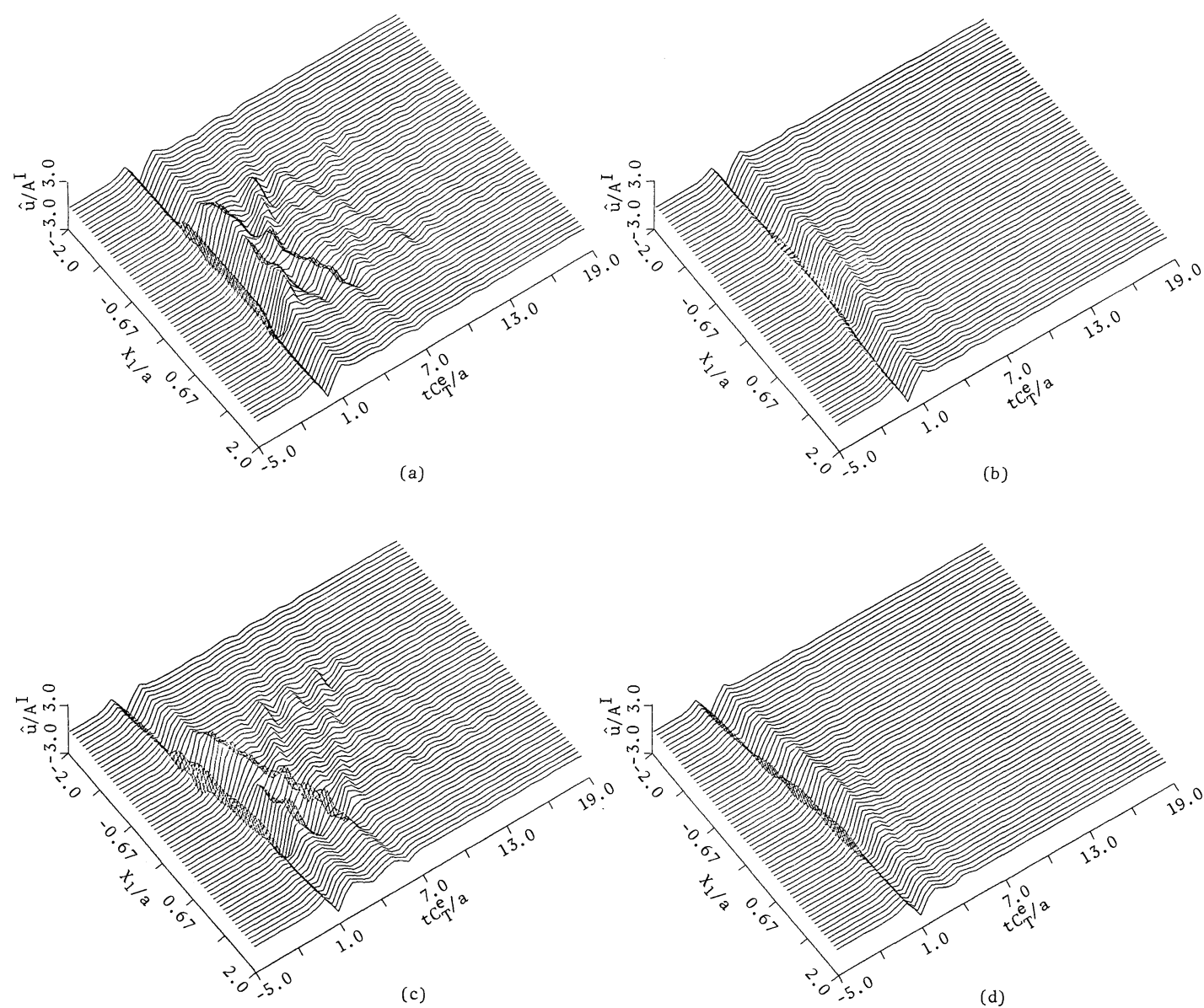

(c)

(d)

Fig. 6 Transient surface motions for the Ricker SH wavelet. Figs. (a) to (d) correspond to those in Fig.5, respectively.

see a lot of ripples which propagate laterally along the surface. These lateral waves are generated due to the surface layer with low velocity, and considered as the local surface waves as pointed out by Bard \& Bouchon $^{20)}$.

\section{CONCLUSIONS}

In the present paper, the surface motions of inhomogeneous alluvial valleys were investigated by the integral equation methods. Three types of integral equations were proposed, each of which was proved to have the sufficient accuracy. Taking account of the efficiency and applicability of each method, Method A with the help of the static fundamental solution was recommended for the analysis of seismic motions of inhomogeneous media. Furthermore, it is possible to apply Method A to inplane problems including inhomgeneous media ${ }^{21)}$, and also to dynamic analysis of anisotropic bodies ${ }^{22}$.

From numerical results, it was shown that the large amplification and the local surface waves were induced due to the soft surface layer of the alluvial valley. In particular, the significant effects of local surface waves were clarified from the point of view of the earthquake engineering, because the surface motion continued to vibrate long after the passage of the incident wave.

\section{APPENDIX The derivation of the fundamental solution $\Gamma$ in eq. (20)}

We define the Fourier transform, the Hankel transform and their inverse transforms as follow, 
Fourier transform:

$\overline{-}(\xi)=\int_{-\infty}^{\infty} \cdot(x) e^{i \xi x} d x$

inverse Fourier transform:

$\cdot(x)=\frac{1}{2 \pi} \int_{-\infty}^{\infty}-(\xi) e^{-i \xi x} d \xi \cdot$

Hankel transform :

$\widetilde{\cdot}(x)=\int_{0}^{\infty} \cdot(\zeta) J_{\nu}(x \zeta) \zeta d \zeta$

inverse Hankel transform:

$\cdot(\zeta)=\int_{0}^{\infty} \sim(x) J_{\nu}(x \zeta) x d x$

If the Fourier transform is applied to eq. (20), we have

$$
\left[\frac{\partial^{2}}{\partial g^{2}}+\frac{1-2 \beta}{g} \frac{\partial}{\partial g}+\left(\frac{\beta^{2}-\nu^{2}}{g^{2}}-\xi^{2}\right)\right] \bar{\Gamma}\left(\xi, g ; Y_{1}, h\right)=-\delta(g-h) e^{i \xi Y_{1}}
$$

where

$$
g=X_{2}+q / p, \quad h=Y_{2}+q / p, \quad \beta=\frac{1+\gamma}{2}, \quad \nu^{2}=\left(\frac{1-\gamma}{2}\right)^{2}-\frac{\left(k_{T}^{0}\right)^{2}}{p^{2}} .
$$

Eq. (A.5) is a kind of Bessel's equation. Substituting the following change of variables into eq. $(\mathrm{A} \cdot 5)$, $\bar{\Gamma}\left(\xi, g ; Y_{1}, h\right)=g^{\beta} \bar{\Phi}\left(\xi, g ; Y_{1}, h\right), \quad \zeta=\xi g$

and then operating the Hankel transform $(\mathrm{A} \cdot 3)$, the transformed solution $\tilde{\bar{\Phi}}$ is obtained as follows,

$$
\tilde{\bar{\Phi}}\left(\xi, \varkappa ; Y_{1}, h\right)=\frac{J_{\nu}(\varkappa \xi h) e^{i \xi Y_{1}}}{\left(\varkappa^{2}+1\right) h^{\beta-1}}
$$

By the application of the inverse transforms $(\mathrm{A} \cdot 4)$ and $(\mathrm{A} \cdot 2)$ to eq. $(\mathrm{A} \cdot 6)$, the fundamental solution $\Gamma(\underset{\sim}{X}$, $\underline{Y})$ in the original space is obtained as

$$
\Gamma(\underset{\sim}{X}, \underset{\sim}{Y})=\frac{1}{2 \pi}\left(\frac{g}{h}\right)^{\beta-1 / 2} Q_{\nu-1 / 2}\left(\frac{r^{2}}{2 g h}+1\right)
$$

where $Q_{\nu}(\cdot)^{\prime}$ denotes the Legendre function of the second kind.

\section{REFERENCES}

1) Kobayashi, S. : Some problems of the boundary integral equation method in elastodynamics, Boundary Elements (Proc. 5th Int. Conf. on BEM in Eng. ), Springer-Verlag, pp. 775 784, 1983.

2) Wong, H. L. and Jennings, P. C. : Effects of canyon topography on strong ground motion, Bull. Seism. Soc. Am., Vol.65, pp. 1239 1257, 1975.

3) Sáchez-Sesma, F. J. and Rosenblueth, E. : Ground motion at canyons of arbitrary shape under incident SH waves, Int. J. Earthq. Eng. Struc. Dyn., Vol. 7, pp. 441 450, 1979.

4) Shah, A. H., Wong, K. C. and Datta, S. K. : Diffraction of plane SH waves in a half space, Int. J. Earthq. Eng. Struc. Dyn., Vol. 10, pp. 519 528, 1982.

5) Niwa, Y. and Hirose, S. : Scattering problems of plane waves in wedge-shaped elastic domains, Theor. Appl. Mech., Vol. 33, Univ. Tokyo Press, pp. 393 406, 1985.

6) Wong, H. L., Trifunac, M. D. and Westermo, B. : Effects of surface and subsurface irregularities on the amplitudes of monochromatic waves, Bull. Seism. Soc. Am., Vol.67, pp. 353 368, 1977.

7) Sánchez-Sesma, F. J. and Esquivel, J. A. : Ground motion on alluvial valleys under incident plane SH waves, Bull. Seism. Soc. Am., Vol. 69, pp. 1107 1120, 1979.

8) Dravinski, M. : Scattering of plane harmonic SH wave by dipping layers or arbitrary shape, Bull. Seism. Soc. Am., Vol.73, pp. 1303 1319, 1983.

9) Toki, K. and Sato, T. : Seismic response analyses of ground with irregular profiles by the boundary element method, Boundary Elements, ibid., pp.699 708, 1983.

10) Kobori, T. and Shinozaki, Y. : Applications of the boundary integral equation method to dynamic soil-structure interaction analysis under topographic site condition, Boundary Elements, ibid., pp.731 740, 1983.

11) Dravinski, M. : Influence of interface depth upon strong ground motion, Bull. Seism. Soc. Am., Vol. 72, pp. 597 614, 1982. 
12) Dravinski, M. : Ground motion amplification due to elastic inclusions in a half-space, Int. J. Earthq. Eng. Struc. Dyn, Vol. 11, pp. 313 335, 1983.

13) Wong, H. L. : Effect of surface topography on the diffraction of P, SV, and Rayleigh waves, Bull. Seism. Soc. Am., Vol.72, pp. 1167 1183, 1982.

14) Niwa, Y., Kitahara, M. and Ikeda, H. : The BIE approach to transient wave propagation problems around elastic inclusions, Theor. Appl. Mech., Vol. 32, pp. 183 198, 1984.

15) Sánchez-Sesma, F. J. : Diffraction of elastic waves by three-dimensional surface irregularities, Bull. Seism. Soc. Am., Vol. 73, pp. 1621 1636, 1983.

16) Kobayashi, S. and Kishima, T. : Dynamic analysis of non-homogeneous ground movements by the boundary integral equation-finite element hybrid method, Numer. Meth. Geomech. (Proc. 5th Int. Conf. Numer. Meth. Geomech.), Balkema, Vol. 1, pp. 135 142, 1985.

17) Niwa, Y., Kitahara, M. and Yamazaki, M. : Resonance analysis of an inhomogeneous protrusion on a semi-infinite stratum, Theor. Appl. Mech., Univ. Tokyo Press, Vol.31, pp. 245 263, 1982.

18) Banerjee, P. K. and Butterfield, R. : Boundary Element Methods in Engineering Science, McGraw-Hill Book Co., Ch. 3, 1981.

19) Trifunac, M. D. : Surface motion of a semi-cylindrical alluvial valley for incident plane SH waves, Bull. Seism. Soc. Am., Vol.61, pp. 1755 1770, 1971.

20) Bard, P. -Y. and Bouchon, M. : The seismic response of sediment-filled valleys. Part 1. The case of incident SH waves, Bull. Seism. Soc. Am., Vol. 70, pp. 1263 1286, 1980.

21) Niwa, Y., Kitahara, M. and Hirose, S. : Elastodynamic problems for inhomogeneous bodies, Boundary Elements, ibid., pp. 751 〜763, 1983

22) Niwa, Y., Kitahara, M. and Hirose, S. : Dynamic analysis of inhomogeneous anisotropic bodies by integral equation method (to appear).

(Received May 10 1985) 